Article

\title{
Gamifying Motor Rehabilitation Therapies: Challenges and Opportunities of Immersive Technologies
}

\author{
Bruno Ferreira *(i) and Paulo Menezes \\ Institute of Systems and Robotics, Department of Electrical and Computer Engineering, University of Coimbra, \\ 3004-531 Coimbra, Portugal; PauloMenezes@isr.uc.pt \\ * Correspondence: bruno.ferreira@isr.uc.pt
}

Received: 31 December 2019; Accepted: 2 February 2020; Published: 7 February 2020

check for updates

\begin{abstract}
Recovering from a traumatic incident (e.g, a stroke) implies rigorous and demanding therapies to ensure recovery of the lost capabilities. Due to the lack of short-term visible results, stroke patients tend to lose interest in their recovery process and frequently do not follow their therapists suggestions to continue performing their training at home, between physiotherapy sessions. This article explores the extent to which common computer games or specifically crafted games can serve as a rehabilitation practice, but also how conventional therapeutic devices might be transformed to be incorporated into games. Furthermore, we propose a platform that follows the opportunity of creating serious games that are designed for stroke patients with reduced upper limb mobility while following the same principles of common therapeutic procedures. This platform was carefully built with the purpose of allowing patients to comply tele-rehabilitation and promoting the execution of the recommended training at home. Following these ideas, we integrated two carefully designed games that have been tested and validated in our previous works, and we added a top-layer characterized by an online back-end application for therapists that allows them to observe their patients' progress over time and draw different conclusions.
\end{abstract}

Keywords: tele-rehabilitation; serious games; engagement; motor rehabilitation; sensory stimulation

\section{Introduction}

The world is now moving at a faster pace than ever before, which means that our current society demands swifter decisions and results from everyone's work or studies. As individuals try to coexist with this fast-paced lifestyle, they tend to neglect their welfare by exposing themselves to higher levels of stress and anxiety, which is known to possibly increase their stroke risk [1]. Besides, there are other different factors that may increase the chances of occurring a traumatic event, such as being overweight or obese; physical inactivity; or even medical risk factors related to high blood pressure, high cholesterol, diabetes, or family history [1,2].

According to the Stroke Association, one in every six people will suffer from a stroke on a global scale, resulting in different types of impairments. Most stroke survivors experience physical disabilities, but other significant consequences can arise in speech, vision, sensory, or cognitive abilities. The success of the stroke recovery process depends on the effective diagnosis, treatment, but most importantly on proper rehabilitation [3]. Even though patients upon leaving the hospital should be in a considered stable condition, they still have a long way to go to ensure recovery of the lost capabilities.

Stroke patients often visit local rehabilitation centers on a daily or weekly basis, where they spend several hours trying to improve functionality and overcome the attained condition. Eighty percent of them exhibit motor deficit, and only approximately $50 \%$ of the stroke survivors are likely 
to regain some functional use of their paretic upper limb [4,5]. Moreover, they have to perform a vast panoply of physical exercises concerning different therapeutic purposes. The most common post-stroke rehabilitation protocols begin with a series of exercises that focus on strengthening the muscles of the arms and shoulders and progressively go towards fine control of hands and fingers.

These exercises are considered to be task-specific, as patients have to perform delimited motions with the purpose of evolving in terms of motor functionality [6]. Given that their execution, besides being painful, can be certainly monotonous, patients soon lose interest in their recovery process. When combined with short-term visible results, there is only a small number of people that try to follow the therapist's instructions in performing those exercises at home beyond physiotherapy sessions. Nonetheless, this has a major impact on the individual's recovery, as more repetitions are known to lead to faster and greater results [3,7].

As technology continues to broaden its horizons while becoming increasingly more accessible for everyone, one can seek a perfect balance between it and the rehabilitation domain. Different types of new tools have been explored throughout the years with the main purpose of aiding stroke rehabilitation, such as robotic devices for motor recovery, brain-computer interfaces (BCI), noninvasive brain stimulators, neuroprostheses, wearable devices for movement analysis, virtual reality (VR), and tablet-pc applications for neurorehabilitation [8]. However, the current popularity of video games among all ages has shown that they can also serve for other purposes rather than pure entertainment, such as therapeutic recovery.

The perfect blend between pedagogical and entertainment aspects can be achieved by using the advances of computer graphics hardware to properly design serious games (SGs). This trendy concept employs gamification principles, frequently used for the construction of the regular video games, to encourage the users to (re)learn something. Although there are many different applications of this significant topic of interest, one can identify several advantages of embracing them during the common rehabilitation therapies.

The work described in this article focuses on VR-based SG addressed to stroke patients with reduced upper limb mobility as a valuable aid for the current motor rehabilitation therapies. With their assistance, users are expected to perform the recommended exercises by their therapists through a playful and enjoyable therapeutic practice. These can be used either at the rehabilitation centers, or at patients' home, stimulating their engagement in the therapeutic process through sets of entertaining games specially customized for the intended purpose.

This article discusses the problem and its inherent opportunities, and presents some pilot results in using VR-based games for post-stroke rehabilitation of the upper limbs. The remainder of the paper is organized as follows. Section 2 presents a background on patient condition and typical therapeutic approaches, the existing adherence issues, and the benefits of SG and game-based therapies. Section 3 presents two different alternatives on how gamification may aid the conventional motor rehabilitation therapies. Section 4 focus on the development of a platform that integrates our previous works on an online back-office for therapists, whereas Section 5 discusses how they can be useful for different recovery stages. Section 6 concludes the article by drawing conclusions from the above and indicate the follow-up work.

\subsection{Related Work}

The idea of providing new ways to perform tele-rehabilitation for stroke patients has been studied and developed by researchers previously to this study. As an example, a web application called "Java Therapy" [9] developed to have stroke survivors performing a customized and specifically tailored program of therapeutic activities. During this therapeutic practice, quantitative feedback of their recovery was given and saved for later analysis. As they evolved throughout sessions, a remote supervising health professional would provide encouragement, but also monitor and alter the exercise program. However, this system was not capable of dynamically adapting himself to the patients' behavior. 
Later, Burke et al. [10] identified two game design principles that should always be considered: meaningful play and challenge. At the start, the game should have a low level of challenge and gradually increase as players become familiar with the game or improve their abilities. A meaningful play emerges from a game in the relationship between a player's actions and the system's outcome.

Ma and Bechkoum have designed a framework for SG movement therapy [11] that included of a number of SG carefully designed and developed to encourage patients to perform physical exercises in adaptive virtual environments (VE). The games were able to adapt accordingly to the users' actions, while having them perform different therapeutic exercises that focused on speed, range of motion, dexterity, and enhancing endurance. These systems also allowed the user performance to be monitored and analyzed over time [12], but could not prevent frustration given the lack of player's capabilities to perform the demanded tasks.

A more recent systematic review on tele-rehabilitation for stroke care was written by Johansson and Wild [13], where the authors reveal promising results to this domain. In fact, the increasing number of implementations and evaluations have increased in the recent years. This suggests that either the supporting technology is achieving a convincing maturity level, or the opportunity and relevance has been recognized by both therapists and game researchers [14]. There is however an attempt to search for new ways of adapting to a broader range of patients, including those not familiar with traditional computer games. For instance, the SG named "CONTRAST" focuses on arm-hand training for stroke survivors using every day physical objects and adapting game's difficulty according to patient's performance [15].

Other works focus on the integration of low-cost systems using state-of-the-art sensors designed for human computer interaction (HCI) or games. As an example, we refer to the work of Sror et al. [16], which makes use of three modules: a passive manipulator that supports horizontal motion against gravity, Kinect and Leap Motion cameras for motion tracking, and a VR game that simulates fish feeding.

On the other hand, training for daily activities can be seen as another trend. "MOVEROffice" is a SG that makes use of VR and natural interaction for upper limbs rehabilitation [17]. The objective is to have patients acquiring skills in performing given tasks in the virtual environment, for later transfer into their daily activities.

\section{Background}

\subsection{Recovering from Stroke and Typical Clinical Condition}

The human brain is a fascinating organ that is in charge of everything that our body does. It keeps supervision of the involuntary activities (e.g., breathing), while providing support for the conscious activities. During a stroke, the blood supply to a certain area of the brain is cut off and brain cells begin to die because of oxygen deprivation. Given the wide variety of the existing body functions, stroke consequences come in many different forms depending on the size and location of the affected brain area. As aforementioned, stroke survivors frequently suffer from physical disabilities, but they can also have experience impairments in cognitive skills, speech, vision, etc.

Upper limb extremities are coordinated by a larger area of the brain, and therefore they are more susceptible to the effects of a stroke [18]. Frequently, stroke survivors struggle to maintain the affected limb stabilized in any position, as a result of weakness or an abnormal state of their muscle tone, described as hypotonia. In these cases, patients are unable to start any type of movement and commonly have their muscles characterized to be in a "floppy" state. This reduced resistance to passive movement is commonly replaced over time by hypertonia, where muscles become surprisingly rigid and exhibit two types of motion resistance: Spasticity, which is related to an increased resistance to passive movement that is velocity-dependent, and rigidity, which is velocity independent, meaning that even if the physiotherapist tries to move the patients arm very quickly, it will not change. Nevertheless, aside from these changes in the muscle tone, it is also frequent to have contractures or simultaneous 
contraction of agonist and antagonist muscles around a joint [18]. These types of abnormal muscular activity can lead to pain and functional disorders, abnormal postures, and stereotyped moves.

Post-stroke patients commonly experience loss or altered sensations as a result of impairments on their somatic sensory side. As a result, they can become unaware of auditory, visual, or tactile stimuli on the contralateral side of the affected area of the brain $[7,18,19]$. Just in terms of visual defects that are commonly reported by them, they may include abnormalities in visual field, eye movements, and vision processing problems [20].

Partial visual field loss is common to $20-30 \%$ of post-stroke patients and means that part of the optical knowledge produced by the retina is not properly received by some layers of the visual cortex [21]. As a frequent result, they are unable to see a section of their visual field and exhibit hemianopia. This phenomenon is identified by the loss of the left and right visual field of both eyes, as shown in Figure 1. Furthermore, other processing issues arise from these blind spots, such as a reduction of both object and people recognition capabilities or even lack of attention triggering [22]. In this sense, patients are unable to detect changes in patterns of visualized motion or even in actions performed in front of them. Some of these visual problems tend to disappear over time, but others require proper therapeutic procedures.
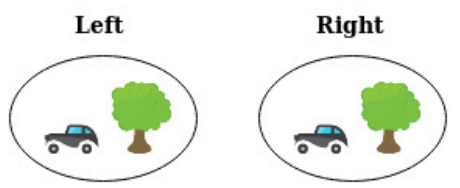

(a)

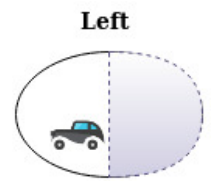

(b)

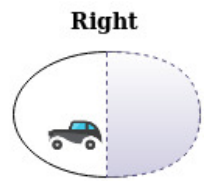

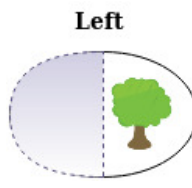

(c)

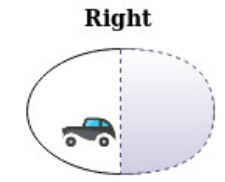

Figure 1. General overview of two different cases of hemianopia when compared to a regular visual field: (a) Normal visual field; (b) Right homonymous hemianopia; (c) Bitemporal hemianopia.

\subsection{Common Therapeutic Practices}

The post-stroke rehabilitation protocols are extremely important to aid patients to improve their quality of life [23]. In fact, upper limb recovery becomes crucial to ensure independence in the performance of the activities of the daily living (ADL), such as drinking from a cup, bathing, or dressing [24]. Although the functional outcome of these therapies is heterogeneous in its nature, its success depends on various factors, such as appropriate timing and the choice of the most suitable rehabilitation program for each individual [25]. Therefore, physiotherapists and occupational therapists will evaluate patients' arm strength, movement range, and sensory capabilities to produce adequate recovery plans based on their needs. These recovery plans comprehend different therapeutic exercises that include practicing custom movements and everyday tasks, where repetition is critical to recover partial or full motor capabilities.

During rehabilitation programs, there are several different therapeutic approaches that physiotherapists may attempt with their patients, while trying to understand if they meet the required needs. Some of them were analyzed and collected from the works French et al. [26], Oujamaa et al. [27], and Sucar et al. [28], presented below.

- Bilateral arm training refers to the use of the non-paretic upper limb to support movements of the affected one, while performing identical activities simultaneously.

- Mirror therapy is described as the process of placing a mirror in a certain condition that reflects the healthy limb as if it was the paretic one. Therefore, it creates the visual illusion of executing normal movements with the paretic limb.

- Occupational therapy focus on aiding patients to recover the ability to perform ADLs, such as dressing or cooking.

- Constraint-induced movement therapy (CIMT) promotes motor (re)learning of the affected upper limb by performing several repetitions of task-specific training while having the healthy one immobilized. 
- Repetitive task training requires having individuals performing a large amount of repetitions for a clear functional objective within a single training session.

- Strength training consists on planning a set of resistance exercises over time to progressively improve muscle strength.

- Splinting or Orthosis techniques are based on the used of external devices as a way to improve functional movement while reducing spasticity or pain. In fact, they might be used to prevent frequent secondary complications, such as contractures.

- For changes in sensation, patients might need to retrain their senses by feeling different materials, objects, or even textures to (re)learn what they feel like.

Considering the aforementioned visual impairments that stroke patients may have, visual scanning training is a commonly used technique that encourages and guides patients to look in a systematic way to both their left and right sides of their visual field. By constantly looking on their blind side, visual processing problems that are related with difficulties of the brain processing the information received by the eyes should disappear over time [29].

\subsection{Adherence Issues}

As stated before, stroke survivors are typically very limited in the range of movements that they are able to perform autonomously, in particular, with the limbs corresponding to the contralateral side of the affected brain area. Post-stroke motor rehabilitation should start as soon as possible, where patients begin with series of exercises that focus on arms and shoulders and progressively go towards fingers and fine control. This early enrollment on motor (re)learning is known to be critical for attaining the best possible recovery outcomes, as it corresponds to the period that the brain has bigger plasticity [23]. Furthermore, principles such as task-specific exercises or performing a enormous amount of repetitions are a transverse and fundamental to all the common practices discussed above.

Despite all the benefits of the common therapeutic practices presented on the above, these tasks are also repetitive across different sessions and boring, as they do not provide any kind of entertainment while sometimes being painful. Although therapists recommend people to continue the execution of exercises at home with the affected upper limb, in most cases these are solely done in the presence of these health professionals. The main reason for this kind of behavior is the fact that there is a lack of short-term or immediate visible results and people gravitate mainly around the long-term possibility of recovery. Therefore, most stroke patients try to overcome their handicap by overusing the healthy limbs to perform the desired ADLs, trying to ignore the affected ones. As they may get progressively efficient in using a single hand and arm based approach for most tasks, the result is that the paretic limb becomes forgotten and later useless. In the opposite side, this might represent some type of a fake reward considering the apparent lack of dependency. However, focusing and forgetting about the affected upper limb is the opposite direction that rehabilitation tries to impose on stroke survivors.

In short, the lack of immediate reward frequently leads to this lack of commitment and interest in continuing the rehabilitation plan at home, which has a direct impact on patients recovery. Therefore, the present work described in this article focuses essentially on new ways to motivate and encourage patients to keep on with the recommended exercises, trying to contribute to reduce dropout and improve recovery success ratios.

\subsection{Advantages of Virtual Reality and Game-Based Therapies}

Considering the adherence issues described above, game-based therapies rely on carefully designed SGs to make the necessary activities of a rehabilitation program easier and more enjoyable than the traditional approaches. In fact, this trendy type of video games has proven successful in the aid of many different areas of application, such as education [30,31], health care [32,33], and even cultural or military training [34,35].

When adding immersive technologies to the equation, VR-based rehabilitation has shown to have the following documented advantages; increased repetitions over the proposed exercises 
and augmented patient engagement; the capacity to track the patient's recovery process over time; possibility of requiring low clinical supervision, which indeed eases home use and tele-rehabilitation; and enhanced motor (re)learning by different types of stimuli (visual, auditory or haptics) $[10,28,36]$. Nevertheless, playing SGs has also shown to be crucial in stimulating the development of basic learning skills, such as paying attention, promoting visual-motor coordination, improving logical spatial reasoning and orientation, among others [37,38].

\subsection{The Sensory Side of Post-Stroke Rehabilitation}

Among the advantages that immersive technologies might provide for therapies, proper sensory information for enhanced motor (re)learning is critical throughout the recovery process [39,40]. When the primary motor cortices are injured by stroke, the human brain is forced to remap its neural activity through mechanisms of neural reorganization and adaption. Researchers believe that there are certain areas of the brain that undergo substantial post-stroke neuroplasticity, such as both ipsilesional and contralesional primary motor cortices and dorsal premotor cortex [41]. Recent studies using neuroimaging techniques show that this remapping process of the brain's sensorimotor interactions leads non-primary motor areas to contribute significantly to the execution of movements using the paretic limb after occurring a stroke. Therefore, patients can regain/relearn motor capabilities over time with proper training by integrating sensory information at the same time [42-44].

Made-up information produced by immersive systems takes this one step further, as it allows us to generate the necessary visual, auditory, and other elements that can be used as stimuli in the "retraining" process [39]. By wearing an HMD and a pair of headphones, we can lead users to perceive that they are performing the desired tasks without actually completing them alone. This creates a controlled environment, where visual game elements and even representations of the player's body may be supervised and adapted to increase self-perception, which helps in the retraining of the visuomotor coordination process. It is however clearly necessary to use instrumented or passive objects that will participate in the haptic stimuli for complete sensory integration. Depending on the characteristics of each patient and extents of the affected zones by stroke, therapists can accordingly choose the parameters of these elements.

\section{Therapies, Games, and Gamification of Therapies: Challenges and Opportunities}

Game-based therapies have grown into a significant area of interest since video games themselves have reached audiences never before imagined. When carefully designed accordingly to the intended purpose, these video games create a sense of engagement, pleasure, and presence that boost every user's motivation that experiences them. Thus, researchers have been focused on how to properly develop new SGs for rehabilitation purposes, without noticing one clear opportunity that therapies might suffer from its benefits. This section explores the extent of how carefully crafted SGs with different therapeutic applications may serve as a valuable aid to stroke rehabilitation. They may be seen as a linking bridge between the current rigorous therapies and a continuous positive attitude throughout every single stage of patients' recovery.

\subsection{How Can a Game Be Used for Rehabilitation?}

In the last decades, some gaming companies have been researching on how to introduce novel ways for players to interact with their consoles and games. This has increased attention on new devices that should replace or simply complement the traditional joysticks and gamepads that traditionally serve as inputs of the users' actions inside the game environment. Nintendo WiiMote, Microsoft Kinect, and Sony Playstation Move are examples of devices that fomented new interaction styles as an attempt to make the user achieve higher levels of immersion when playing games, or even to support new kinds of games that explore the players' body motions as game controls.

Over time, physiotherapists have tried to introduce these devices and its inherent games in the conventional therapies to explore the execution of the required exercises. However, as these 
systems tend to directly map the users' actions into the required game controls, individuals with motor impairments are often asked to perform movements beyond their capabilities. If being unable to produce such movements, they are more likely to fail on the proposed tasks with the consequent negative impact on the users' self esteem. Moreover, when combined with the common depressive states among stroke survivors [2] and the feeling of failure, they are most likely to be conveyed to the path of frustration and quick disapproval of those games. Therefore, this direct usage of commercial games for therapeutic purposes can be considered as the first challenge encountered.

The use of commercial games is still a possibility that is worthwhile and demands to be explored, despite the fact that all of these rely on the conventional interaction systems. Although one cannot expect to find the necessary support for the therapeutic gestures that a patient should execute in most of them, there is always the possibility of discovering a game that can suit certain conditions. Therefore, this game can be appropriate for a given part of the patient's treatment, but it will hardly fulfill the whole range of gestures that should be performed. In our opinion, this approach should not be completely discarded, as good commercial games already contain the most precious ingredient and one clear opportunity, which is the entertainment essence.

Let us think of any video game that is played by just pressing four arrows available on a keyboard or gamepad (up, down, left, and right), or equivalently using a joystick. As it uses simple commands we may raise the question: Would it be possible to turn this game into a therapy? Figure 2 shows three sample exercises that can be performed without the supervision of a therapist, which aim to strengthen patient's muscles of arms and shoulders. It is possible to observe that the performed movements on the left side of the figure are similar to the actions that are required to play the previous game: pressing the left arrow would be equivalent to move the upper extremities to the left, and so on. Therefore, now the question becomes what kind of device can be used as a controller that fulfills the requirements of game and therapy altogether. We may say that almost any motion sensing device can be transformed into a dual-axis controller, from the most complex and expensive such as Polhemus Liberty to Razer Hydra Motion Controllers. Although the former would be more precise, it would require complex calibration and software development. On the other side, the latter, being it already a game controller, may eventually have support on the game itself for mapping the movements into game inputs, requiring only the typical game configuration. If it is not the case, and for the example of personal computer-based games, it is quite simple to develop a simple interfacing application that maps the required movements into keyboard or joystick events. Therefore, adapting the way a patient/player can interact with a carefully chosen game can indeed be seen as one clear opportunity to recycle them to fit therapies' requirements. Nonetheless, we would be taking advantage of the fact that these games were built to be entertaining and pleasing, and focus only on the process of mapping the users' movements into the virtual environments. Yet, it is important to mention that these games should be properly chosen based on the desired actions that are supposed to be performed. For instance, if patients are requested to perform movements in the horizontal plane, from left to right and vice versa; however, if a game is chosen that represent this motion in something moving back and forward, it would clearly mislead our visuomotor coordination, as the visual representation of the motion differs from the actual one.

On the other side, given the vast nature of the existing therapeutic exercises that must be performed at the different recovery stages, one might not find the perfect match between the existing games and the required activities. Additionally, the chosen game must follow one key aspect inherent to every motor rehabilitation therapy, which is repetitive motions. As a higher number of repetitions are proven to be an imperative factor for obtaining better recovery results [18], the game should lead patients to perform cyclical and periodic movements as therapeutically expected. Therefore, discovering a game that guides patients to perform a high amount of repetitions while keeping them engaged can be seen as a major challenge. However, exploring the complete development of SGs for the aid of the existing rehabilitation therapies might be the path to follow. 


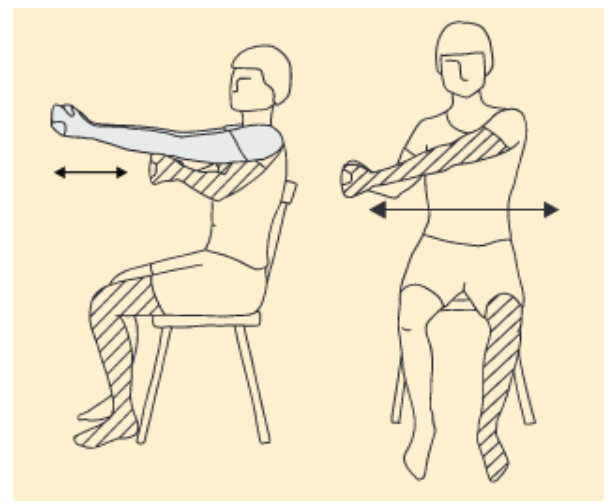

(a)

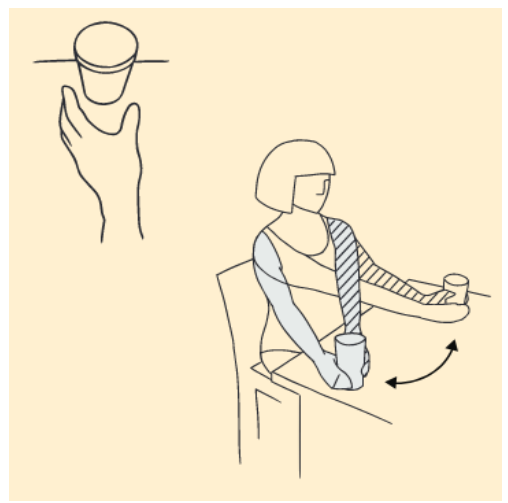

(b)

Figure 2. Three sample exercises that might be performed by patients while sitting. Taken from Your Guide to Exercise after a Stroke available in [45]: (a) Simple exercise while sitting; (b) Exercise for strength and control with both arms on a table.

Specifically crafted games may provide more focused exercises for patient recovery than the commercial ones. However, a survey study created by Hung et al. in [46] shows that therapists report that their patients often describe them as "easy to get bored" or "games are not fun". For that reason, it is mandatory that during the design of these games, special attention is paid to value the entertainment essence while being focused on the desired gestures to be performed by patients.

Here, gamification principles can be used to mitigate these side effects, as it can be described as a technique of bringing game-related elements to non-game contexts. In other words, it can transform repetitive and monotonous tasks into playful experiences, aiding users to become more motivated and engaged with non-game scenarios [47]. To this end, we consider that the following principles must be taken in consideration during the design and creation process.

1. The therapeutic gestures should be perfectly comprised inside the virtual environment.

2. Ability to adapt game's mechanics based on players' motor capabilities by some type of initial calibration.

3. Monitor and record players' performance for later evaluation.

4. Adaptive and progressive change of game's level of challenge.

5. Maintain diversity of contents to accommodate different personal preferences.

6. Provide meaningful cognitive and sensory stimuli during gameplay to allow players to acknowledge both correct and incorrect actions, game's events, etc.

7. Provide positive rewards and reinforcements that inspire players to keep engaged.

Besides these recommended guidelines, Table 1 presents some of the common game elements that should also take part in this process.

Table 1. Common game elements used and their description.

\begin{tabular}{|c|c|}
\hline Game Element & Description \\
\hline Goals / Tasks & Something that players must achieve or work towards. \\
\hline Rules & Express how the game is supposed to be played. \\
\hline User Levels & Encodes patient's proficiency. \\
\hline Feedback & $\begin{array}{l}\text { For every action performed there should be an immediate game's response of success } \\
\text { or failure. }\end{array}$ \\
\hline Point System & Amount of points that should be added to the overall score for each successful action. \\
\hline Leaderboards & $\begin{array}{l}\text { General representation of the highest scores obtained. Allows players to compare their } \\
\text { performances to others. }\end{array}$ \\
\hline Time Pressure & Reducing the amount of time available to complete a task. \\
\hline
\end{tabular}




\subsection{How Can a Rehabilitation Procedure Be Turned into a Game?}

Different types of devices are used to aid the therapeutic processes with different purposes, such as for supporting the affected limbs, constrain the movement of some articulations, guide the movement execution, etc. Their use is typically supervised and in several cases: the role of the therapist is to make sure that the patient keeps up with the exercise by trying to give him/her some motivation. Frustration, pain, and boredom are some of the factors that must be counteracted via constant incitement from the therapist. As these factors influence human emotions, and mood influences behavior, pain sensitivity, challenge acceptance, and performance, the direct consequence is that a happy and positive patient will focus more and for longer periods in therapies, resulting in faster and more profound recovery results.

Contrary to the above, one strategy that may indeed revolutionize the conventional motor rehabilitation therapies relies on taking advantage of the devices already used by physiotherapists and simply making them "smarter" and their usage more attractive. For instance, during physiotherapy sessions, patients use different auxiliary devices to aid the execution of the required tasks, such as supination/pronation with gripper, rope and pulley, shoulder wheel, or pedal exercise bike, as shown in Figure 3. Pedal exercise bikes are commonly used for both the upper and lower limb training given their easy way to reinforce recovery or increasing muscle strength. When used solely for the the upper body, it may be referred as a simple version of the Upper Body Ergometer (UBE). Another device is the shoulder wheel that is used to recover movement amplitude of shoulder joints. For these two types of equipment, one can use simple Bluetooth Cyclometers to provide information about the number or turns per time unit, for example. More complex devices based on optical encoders may acquire information about partial spins when the patient cannot perform complete ones. Furthermore, rope pulleys and supination/pronation grippers can also be similarly instrumented to measure speed and amplitude of movements while performing exercises with them.

These devices already support the constrained execution of the therapeutic movements, but one can see that what is left out of the equation are the motivational elements to execute them. Thus, the gamification of these therapies while incorporating these instrumented devices as interaction inputs may represent the missing part. This ability to turn rehabilitation procedures into games clearly becomes one powerful opportunity that ought to be explored.

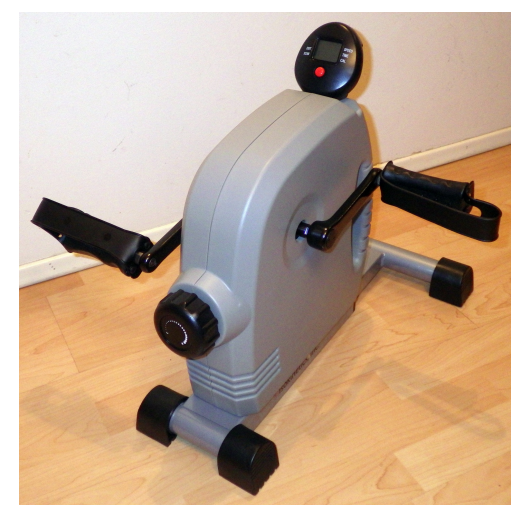

(a)

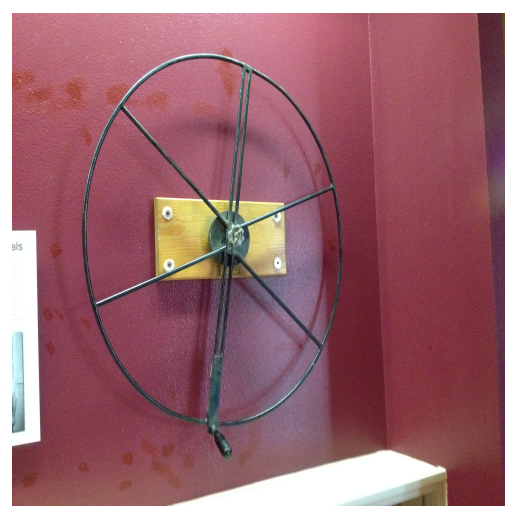

(b)

Figure 3. Cont. 


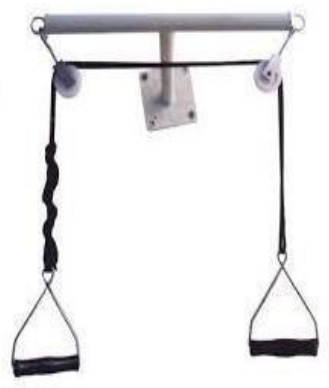

(c)

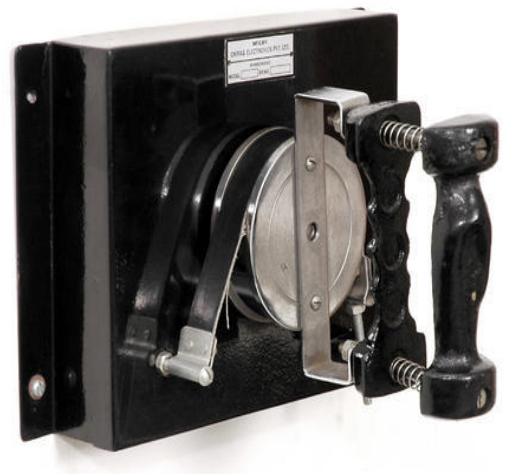

(d)

Figure 3. Examples of equipment used in physiotherapy sessions: (a) Pedal exercise bike; (b) Shoulder wheel; (c) Shoulder Pulley T Type sold by Bharat Bio Products. (d) Supination/Pronation Gripper sold by Chirag Electronics Private Limited.

For instance, the instrumented version of the pedal exercise bike can be used to play a game where the user cruises a virtual city or world. Where one can simply imagine that by placing a regular screen in front of patients and having them wear headphones would enable them to play the crafted SG, while being prone to our crafted stimuli and positive reinforcements. Or even in VR by creating a virtual environment that follows the same principles explained before, where the actions performed by the player in the real world with real objects are transported and translated accordingly into virtual ones. To this end, we would be driving patients' attention to the game itself, instead of having them focused only on the boring repetitions that they are performing.

The proposed design process is illustrated in Figure 4; note that it is divided essentially in two different cores, one for providing the capability of collecting data to the rehabilitation devices and the actual design of a SG. Additionally, the effort still focus on the development of the game, but the method for creating therapeutic movements would be directly obtained from these practices and serve only as a backbone for the final application.

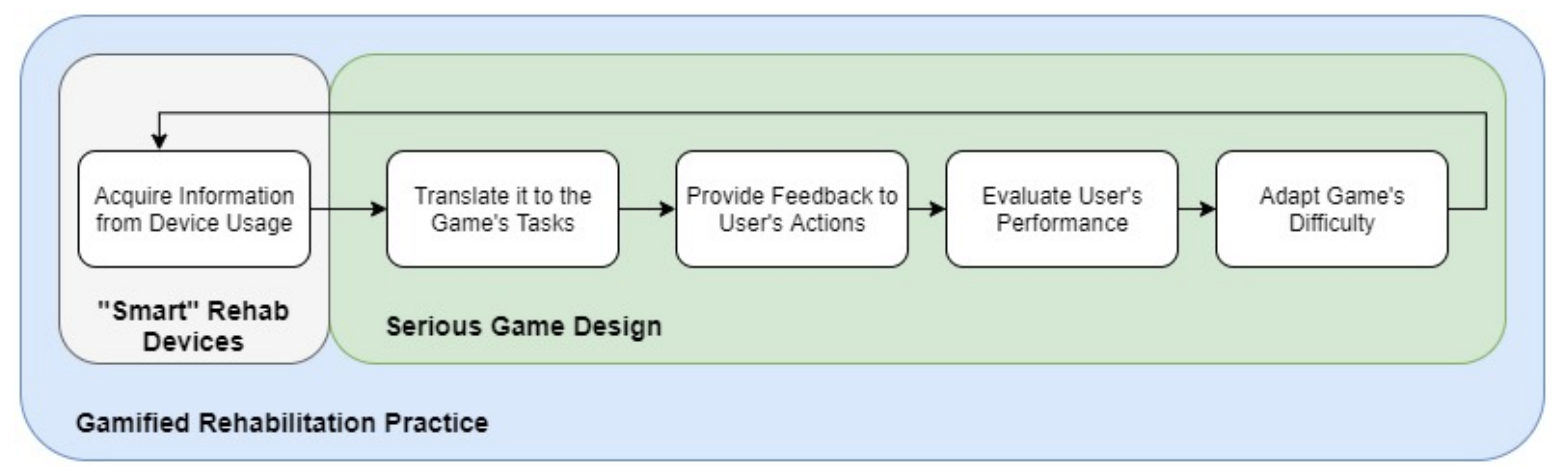

Figure 4. Visual description of the Gamified Rehabilitation Practice approach.

As above stated, the adaptation of existing games via these therapeutic devices transformed into game controllers is a serious possibility. Some examples for games that make use of the used devices in physiotherapy sessions are presented below:

- $\quad$ Pedal exercise bikes - games that simulate activities like canoeing or swimming. For instance, a treasure hunt game, where players navigate in a virtual sea by using their arms or legs motion to propel a boat. The objective is to meet different checkpoints that are spread across a map, which will provide hints for the hidden treasure.

- $\quad$ Shoulder wheel-this physiotherapy device can be used to perform different therapeutic exercises for strengthening the shoulder muscles. When used with both hands, the executed gestures can 
be used for a multiplayer racing car simulator. On the other side, when using it with only one hand for the fully extension of the arm, it can be translated for a firefighter pump game. In this sense, patients would have to supply the water hose for a firefighter companion to prevent a fire.

- Shoulder Pulley T Type-simulating the flapping of a flying bird that is exploring the skies can be an interesting approach for this equipment. Moreover, for initial stages of recovery, where the range of possible movements is reduced, the expected task could be maintaining an hand glider stable while also exploring skies. To this end, the player would have to compensate possible wind flows.

- Supination/Pronation Gripper-similar to the Space Invaders game shown in Figure 5, the patient could use the pronation and supination gesture to control the space ship while avoiding alien missiles. Another example, could be skiing games, such as the Tux Race shown in Figure 5.

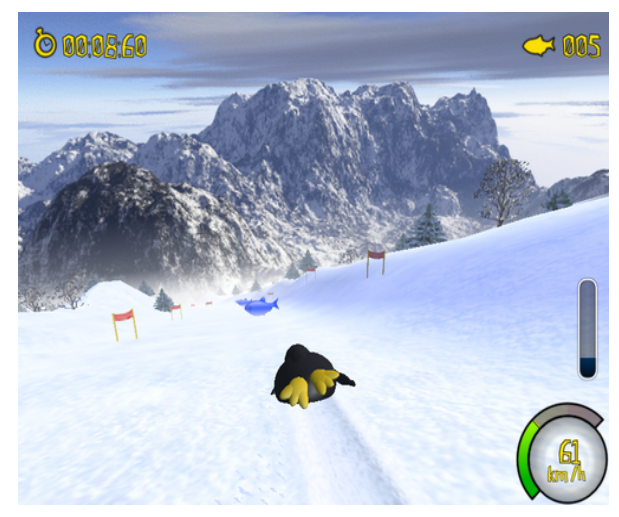

(a)

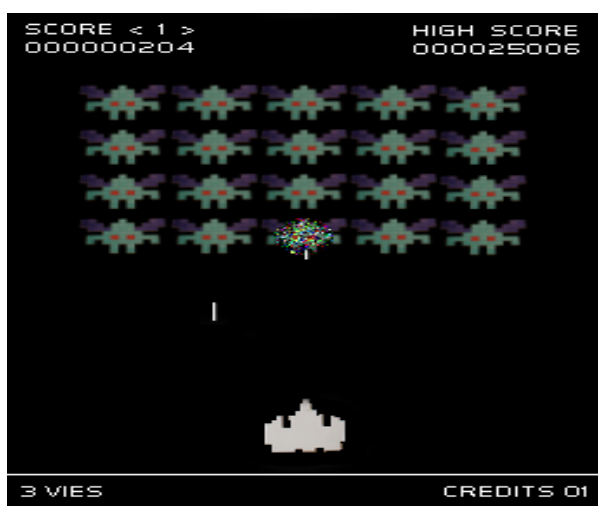

(b)

Figure 5. Screenshots of (a) Tux Racer game created by Sunspire Studios and (b) Space Invaders designed by Tomohiro Nishikado.

As already stated, frequently, physiotherapists have to play the role of a cheering caregiver to provide emotional support, instead of being actually rectifying the subjects' activities. In this situation, all the attention of the physiotherapist is driven to one and only patient. However, this positive reinforcement can be provided by these games, which would give the opportunity to this health professionals to focus only on the task of supervising the patients. Therefore, one possible additional advantage of this approach is that physiotherapists may guide and follow multiple stroke patients performing their rehabilitation simultaneously.

\section{Developing a Platform for Tele-Rehabilitation}

Considering the above, in this section, we present a framework targeting upper limb therapeutic exercises, supporting its use both at patients' homes and at therapists' offices. It also presents an overview on some qualitative parameters we considered as important/interesting for health professionals to analyze the performance and its evolution along the multiple sessions for each individual. A specifically crafted back-office provides the necessary support for storing session data, and for the therapist to either change game parameters or assess patients evolution expressed on the referred parameters. Last, the outline for the created framework and which setup can be used along with our previously developed serious games is also presented.

\subsection{Parameters Collected during the Playful Sessions}

Individuals' performances are commonly quantified based on the achieved score at the end of their playing session. For every player's action during the game, there is a consequence that will either increase or decrease their final score. Aside this universal metric, there are others that may be thought for therapeutic extents given the vast nature of the patients motor and cognitive capabilities. These 
parameters will most likely encode valuable information for later analysis by health professionals to assess the current patient's recovery phase, as described below.

- $\quad$ Score: higher scores can be linked to higher correct responses to the demanding tasks.

- Final Level: higher game levels are certainly related to greater challenges, which require agile visuo-motor coordination and faster outcomes.

- $\quad$ Played Time: time plays an important role for any kind of physical exercise. Under therapeutic meanings, one can acknowledge that having longer sessions implies more repetitions of the recommended training.

- Distance Covered: measuring the distances covered by the affected limbs will in turn explain how much patients used their muscles, with the objective of strengthening them.

- Number of changes in Motion Direction: greater number of motion inversions may suggest inaccuracies in movements or that they were performed without objectives.

- Initial Amplitude of Movements: as a result of a calibration process, this metric can be considered to be an extremely important metric to understand patients' evolution over time.

Nevertheless, if one or more of the above parameters are combined together, the conclusions inferred are as follows.

- Low scores and long played times will surely mean that patients were unable to complete the required tasks, which can lead to quick frustration and disapproval of the game.

- Long distance covered by the affected limb and short number of changes in motion direction means that individuals were most likely performing movements of wider range of amplitude, and thus being in a more advanced stage of recovery. In contrast, if short distances are covered with a high number of changes in motion direction, it means that either they are unable to perform wide gestures or they are inaccurate.

Therefore, while the patient is entertained by playing the game, the above parameters are strongly advised to be collected and stored for later evaluation. Other important information to acquire during the overall system's usage is how many sessions patients played in SG and if they were interrupted. Interrupted sessions allow therapists to understand if the individual felt uncomfortable while playing a specific game, or even if they became "lazy" and did not complete the suggested training.

\subsection{Patients' Tracking Tool: A Back-Office for Therapists}

Given the parameters described in the above, we have developed an online back-office that allows therapists to visualize their patients' progress over time, which means that they can keep track of how often or how good their patients are playing the recommended SGs. In addition, it becomes important to provide a subjective evaluation of the patients' evolution over time, as it displays the parameters that are collected during each playing session.

Figure 6 displays the landing page of this online application, where therapists can perform login and go to their "Therapist Area". This area allows them to view all of their patients that have been using the system and the existing SGs, or even to add a new patient.

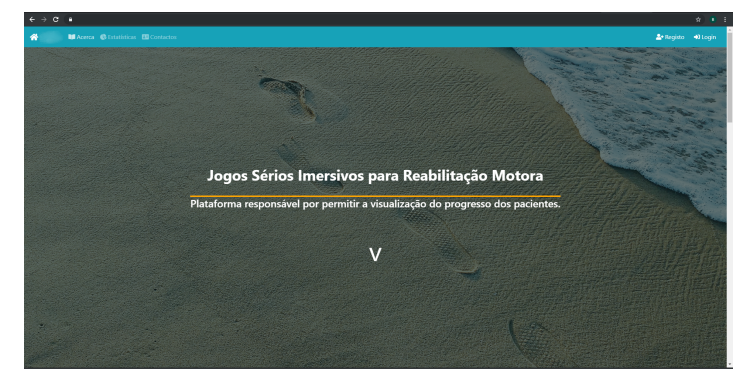

(a)

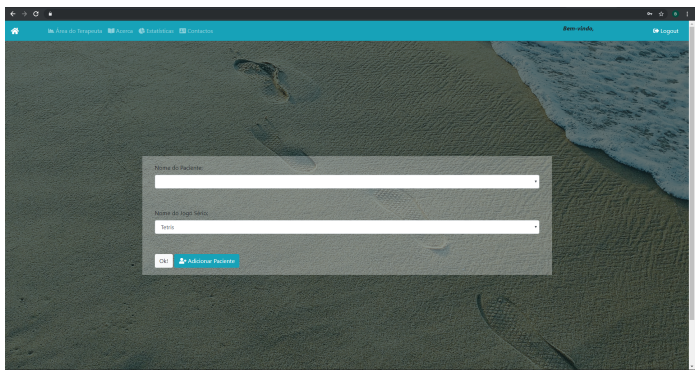

(b)

Figure 6. RehabGames website before and after login: (a) Landing page; (b) Therapist's Area. 
After choosing the desired patient and the SG that they want to visualize, they enter into "Patient's Area", where they can observe the collected parameters through different graphics over different time periods (i.e, 7 days, 30 days, and 1 year), as shown in Figure 7. Furthermore, the back-office also permits therapists to modify each game's parameters (i.e, initial level of difficulty or initial velocity), as well as to change personal information concerning a specific patient. Note that each deployed game across the playing stations fetch the latest changes from the database, which means that if the therapists increases the initial level of difficulty, it will be the starting point for the next session.

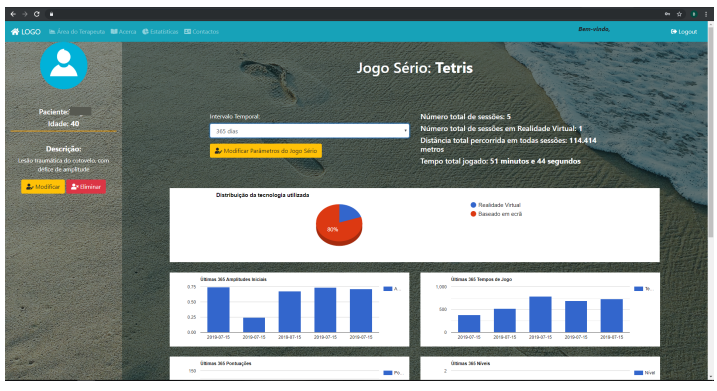

(a)

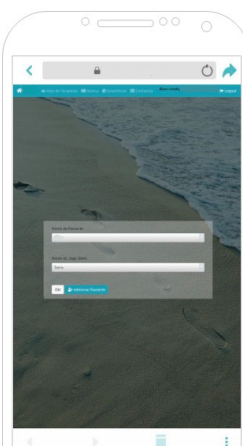

(b)

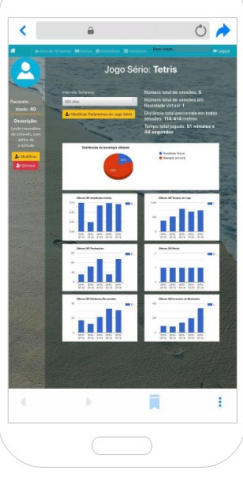

(b)

Figure 7. RehabGames view for laptop and mobile devices: (a) Patient's Area; (b) Mobile view.

\subsection{Platform Outline}

To accomplish a distributed architecture, the developed system is composed of three different fundamental parts: the gaming application, a central database, and the online back-end application for therapists, as shown in Figure 8. One or more play-stations may be created by deploying the gaming application to different laptops or desktops. For instance, one therapist may have more than one of his/her patients training at the same time. Considering that the target audience is mainly composed by older people, the application makes use of a friendly user interface that allows them to set up the playing experience: define which buttons to use, gestures to perform, starting level of difficulty, or whether use the Head-Mounted Display (HMD) or not. While they are playing, the created game dynamically adapts to the users' inputs, and their performances are monitored. Once they finish their session, the collected data concerning usage and evolution of patients are uploaded to the remote database. Subsequently, it becomes automatically available for therapists to consult it whenever desired. If the play-station does not have an internet connection, all the data is saved on a temporary local file and later dispatched to our database.

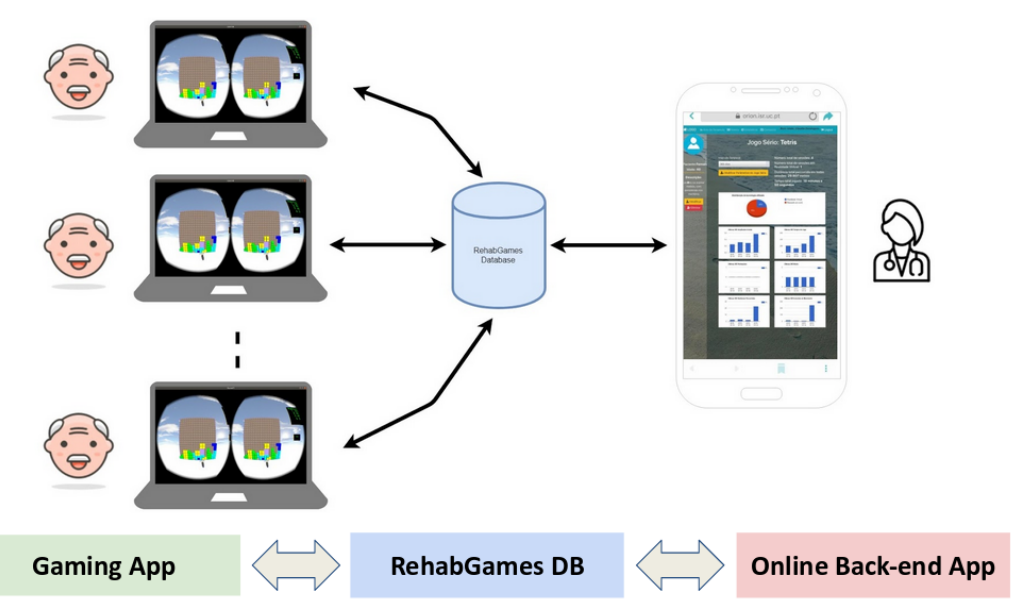

Figure 8. Framework architecture with its three fundamental parts. 


\subsection{Play-Station: Set-Up Required and Currently Available Games}

Each play-station can be used with the help of a regular desktop or laptop, an Oculus Rift, one pair of Razer Hydra Motion Controllers, and some headphones/speakers as shown in Figure 9. The motion controllers are responsible for tracking the user's hand, providing information concerning its position and orientation. It could be performed by several different methods (computer vision techniques, magnetic sensors, or optic flow sensors), but these controllers are easy to move around and comfortable to hold during playtime. The most important aspect is that since different patients have different musculoskeletal conditions/impairments, our created system must be able to understand what is the range of motion that user is capable of performing. To this end, a calibration process under the supervision of therapists was defined, allowing to map the possible movements into the desired ones. Moreover, the available games presented below can be played by a wider range of patients, without leading to quick disapproval or frustration by not being able to complete the demanded tasks given their handicaps. The Oculus Rift DK2 is responsible for displaying the virtual environment to the user, while creating the feeling of presence. Note that the HMD is not suitable for every patient condition and its usage should be advised by the therapist. The use of headphones is recommended with the main purpose of enhancing the feeling of immersion.

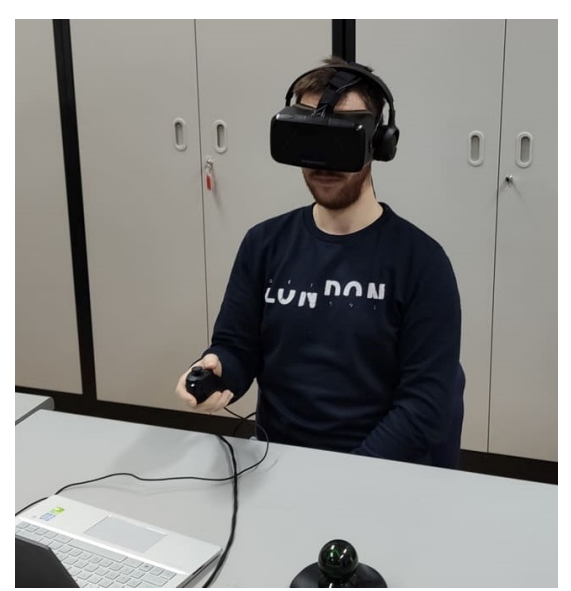

(a)

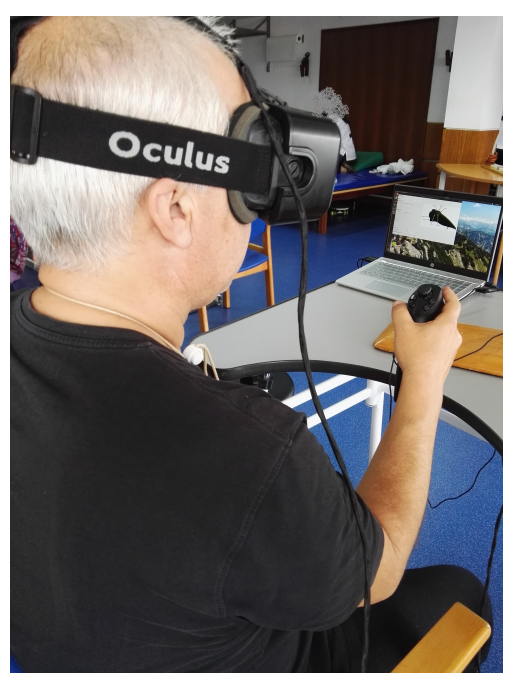

(b)

Figure 9. Two different subjects utilizing the developed platform in distinct places: (a) General set-up required for a play-station; (b) Patient playing one of the developed games in a local rehabilitation center.

\subsubsection{Game 1: Catch \& Dodge}

The objective of Catch $\mathcal{E}$ Dodge game presented in [48] is to catch the higher number of green balls possible during a specific time interval while dodging the red ones. Considering the motor capabilities of the player, these spheres may follow different predefined paths, where the simpler case relies on trajectories for the horizontal plane. However, as patients evolve throughout the sessions and are able to perform a wider range of movements with the corresponding required speed, the difficulty level also increases. In the most simple case, spheres' trajectories come along an horizontal plane that accompanies the inability of most patients that are not able to raise their paretic limb.

Figure 10 shows that the user is represented in the virtual environment as an avatar that may be considered as better representation or healthy version of oneself, as well as the global game scenario with a green sphere approaching him/her. 


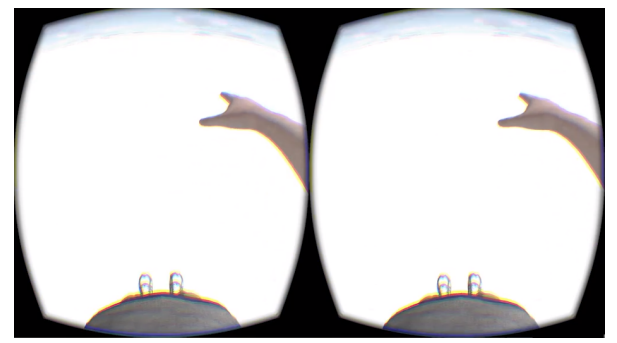

(a)

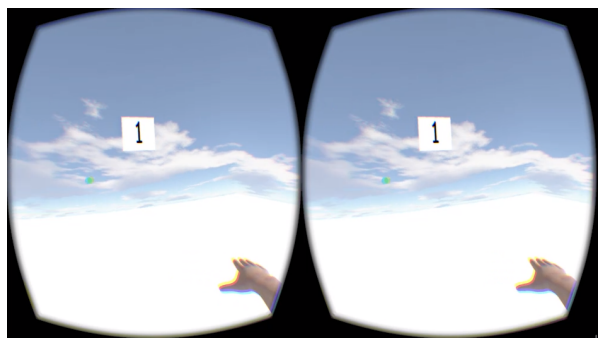

(b)

Figure 10. Gameplay of the developed SG Catch $\mathcal{E}$ Dodge: (a) Initial embodiment process; (b) Green sphere approaching the player.

\subsubsection{Game 2: Tetris4All}

In [49], we proposed an adapted version of the widely known video game Tetris. Its main purpose is to score the maximum amount of points by forming complete horizontal lines on the bottom of a playing area with the falling shapes (tetrominoes). Given the differences across all the stages of stroke patients recovery, this game was carefully designed to have two different playing modes, and therefore named Tetris4All:

- $\quad$ Regular mode: players guide the falling tetrominoes by moving their paretic limb and rotating them if necessary. The rotation process can be achieved by either pressing a button or by rotating their wrist like they were rotating a door knob. As the score increases, the difficulty level also increases, which causes the tetrominoes to fall faster.

- Timed mode: a certain amount of time is given the player to decide the most advantageous position for the tetromino while moving/rotating it on top of the playing area, and when it runs out, the piece falls immediately. The amount of time is calculated for each patient, which means that it enables inexperienced users to have more time to decide where it should fall.

In addition, both these playing modes dynamically adapt to patients motor capabilities, while being designed to enable the execution of movement in the horizontal plane to strengthen their muscles of the arms and shoulders. Moreover, while they are playing their cognitive activity is being stimulated, as Tetris represents a challenge to understanding the interaction between perception, action, cognition, and decision-making [50]. Figure 11 shows a brief overview of the regular mode and one variation that can be applied to the developed SG: bringing the playing area closer to the user's point of view, requires more eye movements to follow tetrominoes, and thus better promoting visual stimulation.

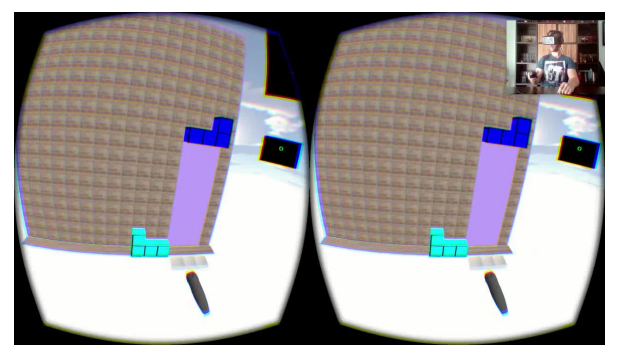

(a)

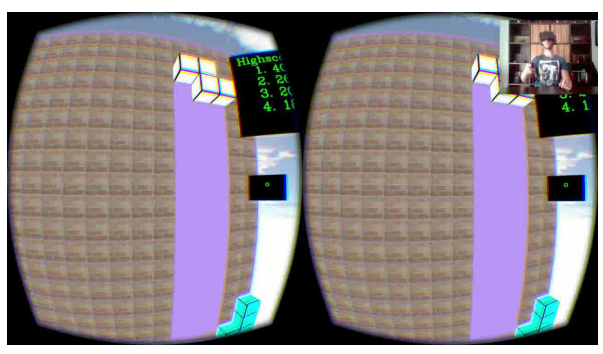

(b)

Figure 11. Gameplay of the developed SG Tetris4All: (a) User playing the regular mode; (b) User playing one possible variation.

\subsection{Implementation Results}

The proposed modified games were carefully designed to follow the same principles as the standard therapeutic practices for the upper limb rehabilitation. As a result of our work, they were presented to therapists that analyzed and gave their approval for the intended purpose. After this initial evaluation, a pilot study around the Tetris game was conducted in order to understand its 
usability in real cases. This study involved nine male and six female participants, with ages of between 30 and 90 years old with the majority (9) of participants in the 50 to 69 range. Further details on the obtained results can be found on our previous work [51].

\section{Discussion}

Developing SGs addressed to stroke rehabilitation means that they must follow the same principles of the established motor rehabilitation therapies, while providing a pleasing experience to the user. Depending on how researchers develop them, they may also serve to simultaneously stimulate both subjects' visual and cognitive activities. As such, one can imagine that if the spheres' trajectories in the Catch $\mathcal{E}$ Dodge game begins on one side and ends at the other, it means that players will cruise around their whole visual field. For the game Tetris 4 All, changing the distance between the playing area and user's point of view will either increase or decrease the difficulty of accompanying tetrominoes while falling. On the other hand, if increasing the velocity of the used objects in the games, players will have less time to make a decision and therefore faster reaction times. Considering that stroke survivors evolve in terms of the amplitude of gestures, perception, visual field and reaction times, the developed SGs must have the ability to internally adapt themselves to players not only during the playing sessions, but also over time.

However, the advantages of using VR and game-based therapies go beyond this, they also allow therapists to encourage patients to perform the typical boring exercises that are recommended at home. Feeding the online back-office application with usage data that was collected during gameplay allows therapists to perform a subjective evaluation on how often and how good their patients are performing the rehabilitation plan between physiotherapy sessions. Moreover, therapists are able to change the initial difficulty level and velocity of each game considering their observations of patients' evolution over time. If players are not having better results throughout several sessions, it means that these parameters are over their motor capabilities, and therefore must be reduced. As stated above, the continued activity at home is known to increase the success of their recovery and thus, producing faster and more visible results.

\section{Conclusions}

This article exposed both challenges and opportunities that occur when trying to gamify the common rehabilitation therapies. We presented different guidelines and common game elements that should be taken into consideration during the design process of a SG for the therapeutic purpose. However, given the difficulties behind creating such a game that complies and intrinsically reproduces the therapeutic gestures, we explored the possibility of using the existing physiotherapy devices to serve as a new way to interact with these games. Therefore, researchers would only focus on how to create motivational games that keep users engaged and committed to their recovery process. Nevertheless, the side of creating new SGs can be explored specifically to guide patients into performing their training at home, which promotes the tele-rehabilitation domain. Therefore, the development of a complete platform for stroke rehabilitation that contains two different validated SGs adapted to various stages of recovery was also presented. Given that this platform was built following a modular design, other developed games with different therapeutic objectives can be easily integrated within it. Moreover, a back-end application for therapists that allows them to track their patients' progress over time was integrated into this platform. As future work, we intend to further explore the adaptation and/or creation of different games that would benefit from the use of these physiotherapy devices for interaction.

Author Contributions: Conceptualization, B.F. and P.M.; Investigation, B.F. and P.M.; Writing—original draft, B.F.; Writing-review \& editing, B.F. and P.M. All authors have read and agreed to the published version of the manuscript.

Funding: This work has been partially supported by OE-National funds of FCT/MCTES (PIDDAC) under project UID/EEA/00048/2019. 
Conflicts of Interest: The authors declare no conflicts of interest.

\section{Abbreviations}

The following abbreviations are used in this manuscript:

$\begin{array}{ll}\text { ADL } & \text { Activities of the Daily Living } \\ \text { BCI } & \text { Brain-Computer Interfaces } \\ \text { CIMT } & \text { Constraint-induced Movement Therapy } \\ \text { HCI } & \text { Human Computer Interaction } \\ \text { HMD } & \text { Head-Mounted Display } \\ \text { SG } & \text { Serious Game } \\ \text { UBE } & \text { Upper Body Ergometer } \\ \text { VE } & \text { Virtual Environment } \\ \text { VR } & \text { Virtual Reality }\end{array}$

\section{References}

1. Graber, M.; Baptiste, L.; Mohr, S.; Blanc-Labarre, C.; Dupont, G.; Giroud, M.; Béjot, Y. A review of psychosocial factors and stroke: A new public health problem. Revue Neurol. 2019, 175, 686-692. [CrossRef] [PubMed]

2. Stroke Association. State of the Nation: Stroke Statistics. 2018. https://www.stroke.org.uk/sites/default/ files/state_of_the_nation_2018.pdf (accessed on 15 September 2019).

3. English, C.; Bowen, A.; Hébert, D.; Bernhardt, J. Rehabilitation after stroke: Evidence, practice, and new directions. In Warlow's Stroke: Practical Management; Hankey, G.J., Macleod, M., Gorelick, P.B., Chen, C., Caprio, F.Z., Mattle, H., Eds.; Wiley Online Library: Hoboken, NJ, USA, 2019; pp. 867-877.

4. Lee, M.M.; Cho, H.; Song, C.H. The mirror therapy program enhances upper-limb motor recovery and motor function in acute stroke patients. Am. J. Phys. Med. Rehabil. 2012, 91, 689-700. [CrossRef] [PubMed]

5. Barker, R.N.; Brauer, S.G. Upper limb recovery after stroke: the stroke survivors' perspective. Disabil. Rehabil. 2005, 27, 1213-1223. [CrossRef] [PubMed]

6. Seidel, P.M.P.; Seidel, G.K. Stroke rehabilitation. In Extracranial Carotid and Vertebral Artery Disease: Contemporary Management; Hans, S.S., Ed.; Springer: Cham, Switzerland, 2018; pp. $279-292$. doi:10.1007/978-3-319-91533-3_24. [CrossRef]

7. Langhorne, P.; Bernhardt, J.; Kwakkel, G. Stroke rehabilitation. Lancet 2011, 377, 1693-1702. [CrossRef]

8. Iosa, M.; Morone, G.; Fusco, A.; Bragoni, M.; Coiro, P.; Multari, M.; Venturiero, V.; De Angelis, D.; Pratesi, L.; Paolucci, S. Seven capital devices for the future of stroke rehabilitation. Stroke Res. Treat. 2012, 2012. doi:10.1155/2012/187965. [CrossRef]

9. Reinkensmeyer, D.J.; Pang, C.T.; Nessler, J.A.; Painter, C.C. Web-based telerehabilitation for the upper extremity after stroke. IEEE Trans. Neural Syst. Rehabil. Eng. 2002, 10, 102-108. [CrossRef]

10. Burke, J.W.; McNeill, M.; Charles, D.; Morrow, P.; Crosbie, J.; McDonough, S. Serious games for upper limb rehabilitation following stroke. In Proceedings of the 2009 Conference in Games and Virtual Worlds for Serious Applications, Coventry, UK, 23-24 March 2009; pp. 103-110.

11. Ma, M.; Bechkoum, K. Serious games for movement therapy after stroke. In Proceedings of the 2008 IEEE International Conference on Systems, Man and Cybernetics, Singapore, 12-15 October 2008; pp. 1872-1877.

12. Rizzo, A.S.; Kim, G.J. A SWOT analysis of the field of virtual reality rehabilitation and therapy. Presence Teleoper. Virtual Environ. 2005, 14, 119-146. [CrossRef]

13. Johansson, T.; Wild, C. Telerehabilitation in stroke care-a systematic review. J. Telemed. Telecare 2011, 17, 1-6. [CrossRef]

14. Bartnicka, J.; Herrera, C.; Michnik, R.; Pavan, E.E.; Vercesi, P.; Varela-Donoso, E.; Garrido, D. The role of virtual reality and biomechanical technologies in stroke rehabilitation. In Advances in Human Factors in Training, Education, and Learning Sciences; Nazir, S., Teperi, A.M., Polak-Sopińska, A., Eds.; Springer: Cham, Switzerland, 2018; pp. 351-361.

15. Jacobs, A.; Timmermans, A.; Michielsen, M.; Plaetse, M.V.; Markopoulos, P. CONTRAST: gamification of arm-hand training for stroke survivors. In Proceedings of the CHI'13 Extended Abstracts on Human Factors in Computing Systems, Paris, France, 27 April 2013; pp. 415-420. 
16. Sror, L.; Levy-Tzedek, S.; Levin, M.F.; Berman, S. Low-Cost Virtual Reality System with Passive Arm Support for Stroke Rehabilitation. Available online: https:/ /www.idiap.ch/workshop/iros2018/files/04frehabsystem21sept2018_iros_final.pdf (accessed on 5 September 2019)

17. Aranha, R.V.; Araújo, L.V.; Monteiro, C.B.M.; Da Silva, T.D.; Nunes, F.L. Moveroffice: Virtual reality for upper limbs rehabilitation. In Proceedings of the 2016 XVIII Symposium on Virtual and Augmented Reality (SVR), Gramado, Brazil, 21-24 June 2016; pp. 160-169.

18. Nordin, K.M.; Chellappan, K.; Sahathevan, R. Upper limb rehabilitation in post stroke patients: Clinical observation. In Proceedings of the 2014 IEEE Conference on Biomedical Engineering and Sciences (IECBES), Kuala Lumpur, Malaysia, 8-10 December 2014; pp. 700-704. doi:10.1109/IECBES.2014.7047597. [CrossRef]

19. Adams, H.P. Principles of Cerebrovascular Disease, 1st ed.; McGraw Hill Professional: New York, NY, USA, 2007.

20. Hepworth, L.R.; Rowe, F.J.; Walker, M.F.; Rockliffe, J.; Noonan, C.; Howard, C.; Currie, J. Post-stroke visual impairment: a systematic literature review of types and recovery of visual conditions. Ophthalmol. Res. Int. J. 2016, 11, 1-43. [CrossRef]

21. Julkunen, L.; Tenovuo, O.; Jääskeläinen, S.; Hämäläinen, H. Rehabilitation of chronic post-stroke visual field defect with computer-assisted training. Restor. Neurol. Neurosci. 2003, 21, 19-28.

22. Rose, F.D.; Brooks, B.M.; Rizzo, A.A. Virtual reality in brain damage rehabilitation. Cyberpsychol. Behav. 2005, 8, 241-262. [CrossRef]

23. Zilli, A. Motor Re-Education of Hemiplegic Patients. Acta Clin. Belg. 1977, 32, $105-111$. doi:10.1080/17843286.1977.11717847. [CrossRef] [PubMed]

24. Hunter, S.M.; Johansen-Berg, H.; Ward, N.; Kennedy, N.C.; Chandler, E.; Weir, C.J.; Rothwell, J.; Wing, A.M.; Grey, M.J.; Barton, G.; et al. Functional strength training and movement performance therapy for upper limb recovery early poststroke-Efficacy, neural correlates, predictive markers, and cost-effectiveness: FAST-INdiCATE Trial. Front. Neurol. 2018, 8, 733. [CrossRef] [PubMed]

25. Rego, P.; Moreira, P.M.; Reis, L.P. Serious games for rehabilitation: A survey and a classification towards a taxonomy. In Proceedings of the 5th Iberian Conference on Information Systems and Technologies, Santiago de Compostela, Spain, 16-19 June 2010; pp. 1-6.

26. French, B.; Thomas, L.H.; Coupe, J.; McMahon, N.E.; Connell, L.; Harrison, J.; Sutton, C.J.; Tishkovskaya, S.; Watkins, C.L. Repetitive task training for improving functional ability after stroke. Cochrane Database Syst. Rev. 2016, 11. doi:10.1002/14651858.CD006073.pub3. [CrossRef] [PubMed]

27. Oujamaa, L.; Relave, I.; Froger, J.; Mottet, D.; Pelissier, J.Y. Rehabilitation of arm function after stroke. Literature review. Ann. Phys. Rehabil. Med. 2009, 52, 269-293. [CrossRef] [PubMed]

28. Sucar, L.E.; Orihuela-Espina, F.; Velazquez, R.L.; Reinkensmeyer, D.J.; Leder, R.; Hernández-Franco, J. Gesture therapy: An upper limb virtual reality-based motor rehabilitation platform. IEEE Trans. Neural Syst. Rehabil. Eng. 2013, 22, 634-643. [CrossRef] [PubMed]

29. Hanna, K.L.; Hepworth, L.R.; Rowe, F.J. The treatment methods for post-stroke visual impairment: A systematic review. Brain Behav. 2017, 7, e00682. [CrossRef]

30. Young, M.F.; Slota, S.; Cutter, A.B.; Jalette, G.; Mullin, G.; Lai, B.; Simeoni, Z.; Tran, M.; Yukhymenko, M. Our princess is in another castle: A review of trends in serious gaming for education. Rev. Educ. Res. 2012, 82, 61-89. [CrossRef]

31. Graafland, M.; Schraagen, J.M.; Schijven, M.P. Systematic review of serious games for medical education and surgical skills training. Br. J. Surg. 2012, 99, 1322-1330. [CrossRef]

32. McCallum, S. Gamification and Serious Games for Personalized Health; pHealth: Quezon, Philippines, 2012; pp. 85-96.

33. Kato, P.M. Video games in health care: Closing the gap. Rev. Gen. Psychol. 2010, 14, 113-121. [CrossRef]

34. Johnson, W.L.; Wang, N.; Wu, S. Experience with serious games for learning foreign languages and cultures. In Proceedings of the SimTecT 2007 Conference, Brisbane, Australia, 4-7 June 2007.

35. Smith, R. The long history of gaming in military training. Simul. Gaming 2010, 41, 6-19. [CrossRef]

36. Lafond, I.; Qiu, Q.; Adamovich, S.V. Design of a customized virtual reality simulation for retraining upper extremities after stroke. In Proceedings of the 2010 IEEE 36th Annual Northeast Bioengineering Conference (NEBEC), New York, NY, USA, 26-28 March, 2010; pp. 1-2. doi:10.1109/NEBC.2010.5458130. [CrossRef] 
37. Bartolomé, N.A.; Zorrilla, A.M.; Zapirain, B.G. Can game-based therapies be trusted? Is game-based education effective? A systematic review of the Serious Games for health and education. In Proceedings of the 16th International Conference on Computer Games (CGAMES). Louisville, KY, USA, 27-30 July 2011; pp. 275-282.

38. Lee, M.M.; Lee, K.J.; Song, C.H. Game-based virtual reality canoe paddling training to improve postural balance and upper extremity function: A preliminary randomized controlled study of 30 patients with subacute stroke. Med. Sci. Monit. 2018, 24, 2590. [CrossRef] [PubMed]

39. Bolognini, N.; Russo, C.; Edwards, D.J. The sensory side of post-stroke motor rehabilitation. Restor. Neurol. Neurosci. 2016, 34, 571-586. [CrossRef] [PubMed]

40. Turville, M.L.; Cahill, L.S.; Matyas, T.A.; Blennerhassett, J.M.; Carey, L.M. The effectiveness of somatosensory retraining for improving sensory function in the arm following stroke: A systematic review. Clin. Rehabil. 2019, 33, 834-846. [CrossRef]

41. Dimyan, M.A.; Cohen, L.G. Neuroplasticity in the context of motor rehabilitation after stroke. Nat. Rev. Neurol. 2011, 7, 76. [CrossRef]

42. Cramer, S.C.; Riley, J.D. Neuroplasticity and brain repair after stroke. Curr. Opin. Neurol. 2008, $21,76-82$. [CrossRef]

43. Hordacre, B.; Moezzi, B.; Ridding, M.C. Neuroplasticity and network connectivity of the motor cortex following stroke: A transcranial direct current stimulation study. Hum. Brain Mapp. 2018, 39, 3326-3339. [CrossRef]

44. Carey, L.; Walsh, A.; Adikari, A.; Goodin, P.; Alahakoon, D.; De Silva, D.; Ong, K.L.; Nilsson, M.; Boyd, L. Finding the Intersection of Neuroplasticity, Stroke Recovery, and Learning: Scope and Contributions to Stroke Rehabilitation. Neural Plast. 2019, 2019. doi:10.1155/2019/5232374. [CrossRef]

45. Margot Andrew, M.H.; Hedges, K. Your Guide to Exercise after a Stroke: A Guide for People with Stroke and Their Families. Available online: https:/ / www.stroke.org.nz/exercise-after-stroke (accessed on 21 November 2019).

46. Hung, Y.X.; Huang, P.C.; Chen, K.T.; Chu, W.C. What do stroke patients look for in game-based rehabilitation: a survey study. Medicine 2016, 95, e3032. [CrossRef]

47. Khaleel, F.L.; Sahari, N.; Wook, T.S.M.T.; Ismail, A. Gamification elements for learning applications. Int. J. Adv. Sci. Eng. Inf. Technol. 2016, 6, 868-874. [CrossRef]

48. Ferreira, B.; Lourenço, J.; Menezes, P. A Serious Game for Post-Stroke Motor Rehabilitation. In Proceedings of the 2019 5th Experiment International Conference (exp. at'19), Funchal, Portugal, 12-14 June 2019; pp. 383-387.

49. Ferreira, B.; Menezes, P. Immersive Serious Games for Post-Stroke Motor Rehabilitation. In Proceedings of the 2019 5th Experiment International Conference (exp. at'19). Funchal, Portugal, 12-14 June 2019; pp. 237-238.

50. Sims, V.K.; Mayer, R.E. Domain specificity of spatial expertise: The case of video game players. Appl. Cogn. Psychol. 2002, 16, 97-115. [CrossRef]

51. Ferreira, B.; Menezes, P. An Adaptive Virtual Reality-Based Serious Game for Therapeutic Rehabilitation. Int. J. Online Biomed. Eng. 2019, Unpublished work.

(C) 2020 by the authors. Licensee MDPI, Basel, Switzerland. This article is an open access article distributed under the terms and conditions of the Creative Commons Attribution (CC BY) license (http:/ / creativecommons.org/licenses/by/4.0/). 